Estudio de caso.

Cómo citar: B. Sanabria y E. Monrroy, “Diferencias cuantitativas entre sistemas constructivos in situ y prefabricados para losas de entrepiso como soporte para la toma de decisiones", Inventum, vol. 13 , no. 25 , pp. 61-72, julio - diciembre, 2018. doi: 10.26620/uniminuto.inventum.13.25.2018. 61-72

Editorial: Corporación Universitaria Minuto de Dios UNIMINUTO.

Recibido: 30 de mayo de 2018 Aceptado: 28 de junio de 2018

Publicado: 3 de septiembre de 2018

Conflicto de intereses: los autores han declarado que no existen intereses en competencia.
Brian Steven Sanabria Riaño bssanabria92@ucatolica.edu.co Universidad Católica de Colombia

Ph.D Edgar Ricardo Monroy Vargas ermonroy@ucatolica.edu.co Universidad Católica de Colombia

\section{DIFERENCIAS CUANTITATIVAS ENTRE SISTEMAS CONSTRUCTIVOS IN SITU Y PREFABRICADOS PARA LOSAS DE ENTREPISO COMO SOPORTE PARA LA TOMA DE DECISIONES}

QUANTITATIVE DIFFERENCES

AMONG IN SITU AND PREFABRICATED CONSTRUCTION SYSTEMS FOR MEZZANINE FLOOR SLABS AS A DECISION-MAKING SUPPORT

DIFERENÇAS QUANTITATIVAS ENTRE SISTEMAS CONSTRUTIVOS IN SITU E PRÉ-FABRICADOS PARA LOUSAS DE ENTREPISO COMO SUPORTE PARA A TOMADA DE DECISÕES

Brian Steven Sanabria Riaño; Ph.D Edgar Ricardo Monroy Vargas

\begin{abstract}
Resumen
En Colombia, son muy pocas las investigaciones académicas que establezcan cuantitativamente la viabilidad en la implementación de soluciones prefabricadas en las construcciones del país y que permitan mejorar la toma de decisiones para el sector productivo. El estado del arte referente al uso de sistemas prefabricados en la construcción se limita esencialmente a un análisis cualitativo, donde únicamente se enumeran las ventajas y desventajas del uso del concreto prefabricado. En el presente documento, se examinan ciertos parámetros dentro de los procesos de diseño y construcción en losas de entrepiso que están presentes y son comunes tanto en sistemas tradicionales como en sistemas prefabricados. Por medio del concepto de cadena de valor de Porter y de la metodología Delphi, se describen y se generan para cada parámetro definido indicadores cuantitativos con el objetivo de generar herramientas que faciliten la toma de decisiones. Dichos indicadores concluirán que es más favorable el uso de soluciones prefabricadas frente a soluciones in situ o tradicionales. Esto supone como panorama positivo la aplicación de dichas soluciones en las construcciones colombianas. Haría falta, entonces, una mayor divulgación y el ajuste de las normas vigentes que le den lugar al prefabricado como un elemento tan confiable como cualquiera de los que se utilizan en la actualidad.
\end{abstract}

Palabras clave: Concreto prefabricado, concreto in situ, construcción, indicador cuantitativo, losa de entrepiso. 


\section{Abstract}

Few academic researches in Colombia establish quantitatively the viabibity of implementing prefabricated solutions in Colombian constructions and contribute to improve the decision-making process for the productive sector. The state of the art of the use of prefabricated systems in construction is basically limited to a qualitative analysis, which only lists the advantages and disadvantages of the use of prefabricated concrete. In this paper, the author examines some patterns in mezzanine floor slabs design and construction, which are commonly used in both traditional and prefabricated systems. Through Porter's concept of value chain and the Delphi methodology, they describe and generate quantitative patterns for each defined parameter in order to create tools to support decision-making. These indicators show that using prefabricated solutions rather than in situ or traditional ones is more convenient. This fact considers the application of such solutions in Colombian constructions as something positive. Therefore, it is necessary to broaden the circulation of knowledge and adjust current rules that place prefabricated concrete as a valuable element, just like any of the ones currently used.

Keywords: Prefabricated concrete, in-situ concrete, construction, quantitative indicator, mezzanine floor slabs.

\section{Resumo}

$\mathrm{Na}$ Colômbia, são muito poucas as investigações acadêmicas que estabeleçam quantitativamente a viabilidade na implementação de soluções pré-fabricadas nas construções do país e que permitam melhorar a tomada de decisões para o setor produtivo. $O$ estado da arte referente ao uso de sistemas pré-fabricados na construção limita-se essencialmente a uma análise qualitativa, onde somente se enumeram as vantagens e desvantagens do uso do concreto pré-fabricado. No presente documento, examinam-se certos parâmetros dentro dos processos de desenho e construção em lousas de entrepiso que estão presentes e são comuns tanto em sistemas tradicionais como em sistemas pré-fabricados. Por médio do conceito de corrente de valor de Porter e da metodologia Delphi, descrevem-se e geram-se para a cada parâmetro definido indicadores quantitativos com o objetivo de gerar ferramentas que facilitem a tomada de decisões. Ditos indicadores concluirão que é mais favorável o uso de soluções pré-fabricadas em frente a soluções in situ ou tradicionais. Isto supõe como panorama positivo a aplicação de ditas soluções nas construções colombianas. Faria falta, então, uma maior divulgação e o ajuste das normas vigentes que lhe dêem local ao pré-fabricado como um elemento tão confiável como qualquer dos que se utilizam na atualidade.

Palavras-chave: Cimento pré-fabricado, concreto in situ, construção, indicador quantitativo, lousa de entrepiso. 


\section{INTRODUCCIÓN}

Según el Consejo Privado de Competitividad (CPC), en los últimos 15 años, el avance en materia de productividad en el país en el sector de la construcción ha sido prácticamente nulo. Por lo anterior, Colombia como un país en vía de desarrollo necesita enfrentarse al reto de modernizarse. La innovación es la clave para construir, no solo edificaciones, sino ciudades de calidad y este debe ser un objetivo claro de los ingenieros civiles [1]. Uno de los llamados de atención hecho a las empresas constructoras del país por parte de Camacol en una de sus últimas reuniones es el compromiso con la inversión en innovación y tecnología; de no hacerlo, multinacionales de la construcción que están haciendo presencia en el país se harán con buena parte de los próximos negocios del sector [2].

Sin duda, para ser competitivos ante las demandas mundiales, se deben reducir y cumplir los plazos contractuales con innovación tecnológica e industrialización de los procesos, pero sin sacrificar los costos y la calidad de las edificaciones, y las empresas de prefabricados estén mejor preparadas para proporcionar apoyo en la entrega a tiempo de los proyectos [3]. En el mundo, la construcción con elementos de concreto prefabricado transformó la lenta y artesanal construcción tradicional [4]; sin embargo, en Colombia las empresas constructoras se muestran escépticas ante la utilización de nuevas formas de industrialización y, por tanto, a la prefabricación: pocas alternativas, mala calidad, costos elevados, entre otras, son las características que se le atribuyen a la construcción prefabricada, para así, mantener las tecnologías de construcción actuales. Sí bien es cierto que existen en el país empresas prefabricadoras intentando permear el mercado, el desconocimiento de las implicaciones (diseño, costos, tiempos, etc.) de usar prefabricados en la construcción de edificaciones no ha hecho posible su implementación en gran escala.

Luego de consultado el estado del arte referente al uso de estructuras de concreto prefabricado en Colombia, llama la atención la poca investigación académica disponible en el país. Salvo algunos trabajos de grado, las publicaciones existentes solo ofrecen una descripción cualitativa en la que se citan las propiedades y las enormes ventajas de los elementos de concreto prefabricado; sin embargo, dichas publicaciones mantienen la incertidumbre en cuanto a costos, tiempos, procesos, etc. Por lo anterior, diseñadores y constructores prefieren mantener sus métodos tradicionales, evitando asumir riesgos que, presumen, pueden tener con la implementación de soluciones industrializadas.

De acuerdo con los argumentos anteriores, utilizando como caso de estudio el diseño y construcción del sistema de entrepiso de una edificación construida en la ciudad de Bogotá, se generan indicadores cuantitativos que permitan establecer las diferencias entre elementos prefabricados y construidos in situ y, así, evaluar qué sistema presenta mayor ventaja. Se analiza técnica y económicamente la implementación del uso de prefabricados y cómo este puede ser una respuesta de innovación y de mejora en costos, procesos y tiempos en un proyecto de construcción tradicional.

Se estudian de manera general los métodos y componentes más usados en el mundo, sus funciones, aplicaciones, mano de obra, equipos requeridos y su aporte al medio ambiente y a la sustentabilidad [5].

Únicamente se tratará lo correspondiente a la estructura de las losas de entrepiso en las edificaciones. Análisis de otros referentes elementos estructurales o actividades como acabados, urbanismo, instalaciones técnicas, etc., no se encuentran definidos dentro del alcance de esta investigación.

\section{DESARROLLO Y DESCRIPCIÓN DE LA METODOLOGÍA USADA}

\section{Descripción del caso de estudio}

Para lograr la comparación entre los procesos de diseño y construcción de los sistemas in situ y prefabricado, se analizan con un ejemplo práctico las variables que intervienen en un proyecto construido con losas de entrepiso prefabricadas tipo losa alveolar y losa maciza (caso A) y el mismo proyecto, pero construido con métodos tradicionales, tipo losa de entrepiso in situ nervada o aligerada (caso B).

El desarrollo del caso de estudio se basa en un proyecto que se construye dentro de un importante centro empresarial ubicado en la calle 26, en el occidente de la ciudad de Bogotá. La edificación consta de tres niveles y un sótano. La estructura la componen columnas, vigas y un muro de contención perimetral en el sótano. Las alturas libres son de 3,54 m en sótano, 4,40 $\mathrm{m}$ en el nivel 1 y 3,80 $\mathrm{m}$ en los niveles 2 y 3 . La superficie aproximada del piso tipo es de $2.600 \mathrm{~m}^{2}$. 
La estructura corresponde a un sistema de pórtico (vigas-columnas) de concreto prefabricado, que resiste las cargas gravitacionales de la estructura, en conjunto con muros de concreto fundido en sitio que aportan la resistencia sísmica del edificio.

Las dimensiones de los principales elementos resistentes son: columnas de $50 \times 50 \mathrm{~cm}$, vigas prefabricadas pretensadas de $45 \times 60 \mathrm{~cm}$ y de $50 \times 60 \mathrm{~cm}$ para los pisos superiores, y vigas prefabricadas sin pretensado de $45 \times 80 \mathrm{~cm}$ más vigas in situ $30 \times 27$ $\mathrm{cm}$ para el cielo sótano.

\section{Metodología de evaluación del caso de estudio}

La evaluación se realiza a partir de la definición de cadena valor de Porter. Esta consiste en una herramienta gerencial para la toma de decisiones y busca caracterizar fuentes de ventaja competitiva, identificando condiciones favorables o desfavorables dentro de un proceso en específico [6].

Una cadena de valor está integrada por actividades primarias o fundamentales y secundarias o de apoyo. Para el desarrollo del presente trabajo, se clasificarán los aspectos mencionados anteriormente, dentro de los procesos de diseño y construcción y, a su vez, estos aspectos se diferenciarán entre primarios o secundarios. Serán primarios si sus resultados se consideran esenciales dentro de la ejecución de un proyecto y serán secundarios aquellos que sirvan de apoyo a los procesos fundamentales.

Dicho lo anterior, se examinan ciertos parámetros dentro de los procesos de diseño y construcción de losas de entrepiso, que están presentes y son comunes en ambos casos estudiados y que, además, sirven de apoyo para la toma de decisiones en el momento de elegir entre un sistema constructivo u otro.

Se delimitan dos etapas generales como son el diseño y la construcción. A partir de estas, se indican a continuación los parámetros de análisis que permitirán concluir si un caso u otro presenta resultados positivos o negativos dentro del caso de estudio.

1. Peso de la estructura

2. Calidad de los materiales

3. Cantidad de materiales

4. Costo directo

5. Duración de actividades

6. Control de desperdicios
Con el fin de establecer parámetros de importancia entre los parámetros que se van a evaluar, se acude al método Delphi, que es una técnica de obtención de información sustentada en la consulta a expertos de un área específica, con el fin de obtener la opinión de consenso más fiable del grupo consultado. Esta técnica, de carácter cualitativo, es usada cuando no se cuenta con información suficiente para la toma de decisiones, ante situaciones de incertidumbre o cuando se carece de información objetiva [7]. En el marco del seminario Usos y Aplicaciones de los Prefabricados de Concreto, organizado por Asocreto, a partir del conversatorio sobre el desarrollo de los prefabricados en Suramérica, entre el ingeniero Gabriel Santana, presidente de la Anippac, y el arquitecto José María Benito, gerente de Suramérica del Grupo Preansa, y basados además en el concepto de cadena de valor, se establecen en una escala de 0 a $100 \%$ los siguientes parámetros de importancia a los indicadores para evaluar con el objetivo de lograr conclusiones más precisas:

Figura 1. Cadena de valor de aspectos para evaluar

\begin{tabular}{|c|c|c|}
\hline \multirow{3}{*}{$\begin{array}{l}\text { ACTIVIDADES } \\
\text { SECUNDARIAS }\end{array}$} & $\begin{array}{l}\text { Indicador Calidad de los } \\
\left.\text { materiales ( } \mathrm{I}_{\mathrm{CM}}\right)\end{array}$ & $13.3 \%$ \\
\hline & $\begin{array}{l}\text { Indicador Cantidad de } \\
\text { Concreto }\left(I_{\infty}\right)\end{array}$ & $13.3 \%$ \\
\hline & $\begin{array}{l}\text { Indicador Sustentabilidad } \\
\text { (Control de desperdicios) ( }\left(I_{\mathrm{CD}}\right)\end{array}$ & $13.3 \%$ \\
\hline \multirow{3}{*}{$\begin{array}{l}\text { ACTIVIDADES } \\
\text { PRIMARIAS }\end{array}$} & \begin{tabular}{|l} 
Indicador Peso de la \\
Estructura $\left(\mathrm{I}_{\mathrm{PE}}\right)$ \\
\end{tabular} & $20.0 \%$ \\
\hline & $\begin{array}{l}\text { Indicador Costo Directo } \\
\left(\mathrm{I}_{\mathrm{APU}}\right)\end{array}$ & $20.0 \%$ \\
\hline & $\begin{array}{l}\text { Indicador Programa de } \\
\text { obra }\left(I_{\mathrm{po}}\right)\end{array}$ & $20.0 \%$ \\
\hline
\end{tabular}

Fuente: Elaboración propia.

\section{RESULTADOS Y DISCUSIÓN}

\section{Resultados del caso de estudio}

\section{Peso de la estructura}

Se tiene únicamente en cuenta el peso propio de cada uno de los elementos de losa para cada caso, losa alveolar, loseta prefabricada y capa de compresión para el caso A, y viguetas y loseta superior para el caso B. 
Figura 2. Comparación del peso entre casos

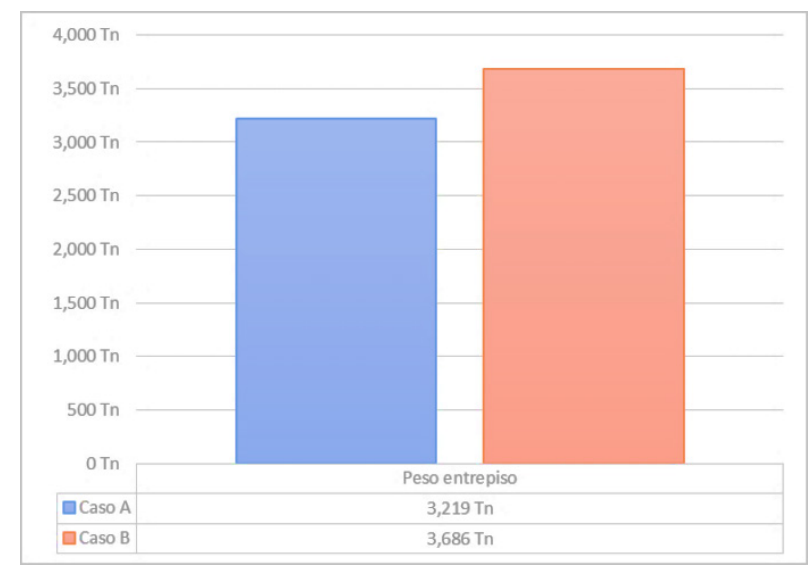

Fuente: Elaboración propia.

De acuerdo con lo anterior, se evidencia que la estructura de entrepiso del caso B representa un $15 \%$ de mayor peso frente al caso $A$.

Tabla 1. Diferencia de peso entre cada caso

\begin{tabular}{|c|c|c|c|c|}
\hline Item & Caso A & Caso B & Diferencia & $\%$ \\
\hline Peso entrepiso & $3,219 \mathrm{Tn}$ & $3,686 \mathrm{Tn}$ & $467 \mathrm{Tn}$ & $15 \%$ \\
\hline
\end{tabular}

Fuente: Elaboración propia.

\section{Calidad de los materiales}

La producción de elementos prefabricados se realiza bajo estrictos controles de calidad. Las fábricas de prefabricados tienen la posibilidad de elaborar sus propios concretos. Este proceso dentro de su plan de calidad permite, además, alcanzar resistencias a la compresión mayores a las de un concreto elaborado en obra e, incluso, a las que se consiguen en el mercado.

Se presenta a continuación la comparación de resistencia a la compresión del concreto para cada caso.

Figura 3. Comparación de f'c entre casos

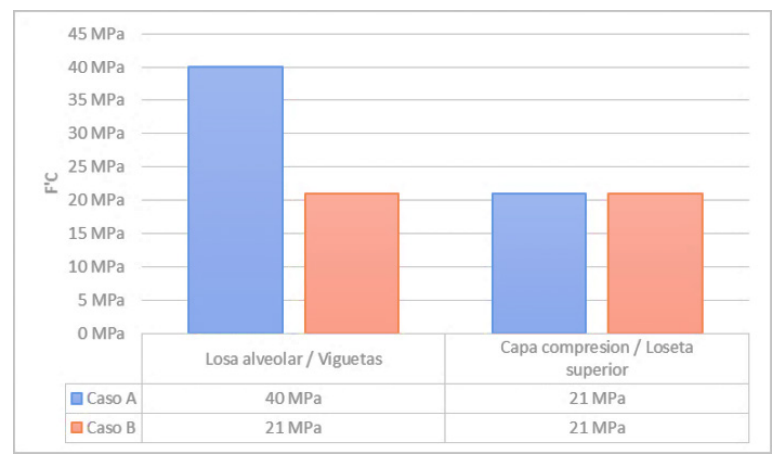

Fuente: Elaboración propia.
Tabla 2. Diferencia de f'c en cada caso

\begin{tabular}{|c|c|c|}
\hline Caso & $\begin{array}{c}\text { Losa alveolar / } \\
\text { Viguetas }\end{array}$ & $\begin{array}{c}\text { Capa compresion } \\
\text { / Loseta superior }\end{array}$ \\
\hline Caso A & $40 \mathrm{MPa}$ & $21 \mathrm{MPa}$ \\
\hline Caso B & $21 \mathrm{MPa}$ & $21 \mathrm{MPa}$ \\
\hline Diferencia & $-19 \mathrm{MPa}$ & $0 \mathrm{MPa}$ \\
\hline$\%$ & $-48 \%$ & $\mathbf{0 \%}$ \\
\hline
\end{tabular}

Fuente: Elaboración propia.

La resistencia a la compresión de los elementos estructurales, viguetas para el caso $\mathrm{B}$, es $48 \%$ menor frente a la losa alveolar y loseta prefabricada para el caso A.

La capa de compresión in situ del caso A, requerida para lograr la transferencia de fuerzas horizontales por la acción del diafragma, se diseña con una f'c a los 28 días de $21 \mathrm{MPa}$. Teniendo en cuenta que es un concreto que se elaborará en obra o se comprará a un proveedor, lo anterior no representa ninguna diferencia frente a la f'c de diseño de la loseta o torta superior del caso B.

\section{Cantidad de concreto}

Una consecuencia del uso de concretos de alto desempeño (f'c entre $40 \mathrm{MPa}$ y $100 \mathrm{MPa}$ ) es la optimización de las secciones de los elementos estructurales, lo cual se traduce en una disminución de la cantidad de concreto.

El análisis de cantidad de volumen de concreto se hizo para resultados teóricos y no se tuvieron en cuenta porcentajes de desperdicio o factores de pérdida de material.

Tabla 3. Resumen volumen de losa prefabricada caso A

\begin{tabular}{|c|c|c|c|}
\hline Item & $\begin{array}{c}\text { Área en } \\
\text { planta }\end{array}$ & $\begin{array}{c}\text { Seccion } \\
\text { transversal }\end{array}$ & $\begin{array}{c}\text { Losa } \\
\text { prefabricada }\end{array}$ \\
\hline Sótano & $3,277 \mathrm{~m} 2$ & $0.11 \mathrm{~m} 3 / \mathrm{m} 2$ & $374 \mathrm{~m} 3$ \\
\hline Piso 1 & $1,756 \mathrm{~m} 2$ & $0.08 \mathrm{~m} 3 / \mathrm{m} 2$ & $141 \mathrm{~m} 3$ \\
\hline Piso 2 & $1,756 \mathrm{~m} 2$ & $0.08 \mathrm{~m} 3 / \mathrm{m} 2$ & $141 \mathrm{~m} 3$ \\
\hline Piso 3 & $1,756 \mathrm{~m} 2$ & $0.08 \mathrm{~m} 3 / \mathrm{m} 2$ & $141 \mathrm{~m} 3$ \\
\hline \multicolumn{3}{|r|}{ Volumen Total } & $796 \mathrm{m3}$ \\
\hline \multicolumn{3}{|c|}{ Area total entrepiso } & $8,547 \mathrm{~m} 2$ \\
\hline \multicolumn{3}{|r|}{ Volumen / m2 } & $0.09 \mathrm{~m} 3 / \mathrm{m} 2$ \\
\hline
\end{tabular}

Fuente: Elaboración propia. 
Tabla 4. Resumen volumen capa de compresión caso A

\begin{tabular}{|c|c|c|c|}
\hline Item & $\begin{array}{c}\text { Área en } \\
\text { planta }\end{array}$ & $\begin{array}{c}\text { Seccion } \\
\text { transversal }\end{array}$ & $\begin{array}{c}\text { Capa de } \\
\text { compresion }\end{array}$ \\
\hline Sótano & $3,277 \mathrm{~m} 2$ & $0.07 \mathrm{~m} 3 / \mathrm{m} 2$ & $229 \mathrm{~m} 3$ \\
\hline Piso 1 & $1,756 \mathrm{~m} 2$ & $0.05 \mathrm{~m} 3 / \mathrm{m} 2$ & $88 \mathrm{~m} 3$ \\
\hline Piso 2 & $1,756 \mathrm{~m} 2$ & $0.05 \mathrm{~m} 3 / \mathrm{m} 2$ & $88 \mathrm{~m} 3$ \\
\hline Piso 3 & $1,756 \mathrm{~m} 2$ & $0.08 \mathrm{~m} 3 / \mathrm{m} 2$ & $141 \mathrm{~m} 3$ \\
\hline \multicolumn{3}{|r|}{ Volumen Total } & $546 \mathrm{~m} 3$ \\
\hline \multicolumn{3}{|c|}{ Area total entrepiso } & $8,547 \mathrm{~m} 2$ \\
\hline \multicolumn{3}{|r|}{ Volumen / m2 } & $0.06 \mathrm{~m} 3 / \mathrm{m} 2$ \\
\hline
\end{tabular}

Fuente: Elaboración propia.

El volumen total de concreto requerido para en el caso A, resultante de la suma del componente prefabricado $\left(796 \mathrm{~m}^{3}\right)$ y la capa de compresión -topping- $\left(546 \mathrm{~m}^{3}\right)$, es de $1,341 \mathrm{~m}^{3}$.

Tabla 5. Resumen volumen viguetas caso B

\begin{tabular}{|c|c|c|c|}
\hline Item & $\begin{array}{c}\text { Área en } \\
\text { planta }\end{array}$ & $\begin{array}{c}\text { Seccion } \\
\text { transversal }\end{array}$ & Viguetas \\
\hline Sótano & $475 \mathrm{~m} 2$ & $0.75 \mathrm{m3} / \mathrm{m} 2$ & $356 \mathrm{~m} 3$ \\
\hline Piso 1 & $254 \mathrm{~m} 2$ & $0.65 \mathrm{~m} 3 / \mathrm{m} 2$ & $165 \mathrm{~m} 3$ \\
\hline Piso 2 & $254 \mathrm{~m} 2$ & $0.65 \mathrm{~m} 3 / \mathrm{m} 2$ & $165 \mathrm{~m} 3$ \\
\hline Piso 3 & $254 \mathrm{~m} 2$ & $0.65 \mathrm{~m} 3 / \mathrm{m} 2$ & $165 \mathrm{m3}$ \\
\hline \multicolumn{3}{|r|}{ Volumen Total } & $852 \mathrm{m3}$ \\
\hline \multicolumn{3}{|c|}{ Area total entrepiso } & $8,547 \mathrm{~m} 2$ \\
\hline \multicolumn{3}{|c|}{ Volumen / m2 } & $0.10 \mathrm{~m} 3 / \mathrm{m} 2$ \\
\hline
\end{tabular}

Fuente: Elaboración propia.

Tabla 6. Resumen volumen loseta superior caso B

\begin{tabular}{|c|c|c|c|}
\hline Item & $\begin{array}{c}\text { Área en } \\
\text { planta }\end{array}$ & $\begin{array}{c}\text { Seccion } \\
\text { transversal }\end{array}$ & $\begin{array}{l}\text { Loseta } \\
\text { superior }\end{array}$ \\
\hline Sótano & $3,277 \mathrm{~m} 2$ & $0.08 \mathrm{~m} 3 / \mathrm{m} 2$ & $262 \mathrm{m3}$ \\
\hline Piso 1 & $1,756 \mathrm{~m} 2$ & $0.08 \mathrm{~m} 3 / \mathrm{m} 2$ & $141 \mathrm{~m} 3$ \\
\hline Piso 2 & $1,756 \mathrm{~m} 2$ & $0.08 \mathrm{~m} 3 / \mathrm{m} 2$ & $141 \mathrm{~m} 3$ \\
\hline Piso 3 & $1,756 \mathrm{~m} 2$ & $0.08 \mathrm{~m} 3 / \mathrm{m} 2$ & $141 \mathrm{~m} 3$ \\
\hline \multicolumn{3}{|r|}{ Volumen Total } & $684 \mathrm{~m} 3$ \\
\hline \multicolumn{3}{|c|}{ Area total entrepiso } & $8,547 \mathrm{~m} 2$ \\
\hline \multicolumn{3}{|c|}{ Volumen / m2 } & $0.08 \mathrm{~m} 3 / \mathrm{m} 2$ \\
\hline
\end{tabular}

Fuente: Elaboración propia.

El volumen total de concreto requerido para en el caso $B$, resultante de la suma de las viguetas $(852$ $\mathrm{m}^{3}$ ) más la loseta superior $\left(684 \mathrm{~m}^{3}\right)$, es de $1,536 \mathrm{~m}^{3}$.
Tabla 7. Diferencia de volumen de concreto en cada caso

\begin{tabular}{|c|c|}
\hline Caso & $\begin{array}{c}\text { Volumen de } \\
\text { concreto }\end{array}$ \\
\hline Caso A & $1,341 \mathrm{~m} 3$ \\
\hline Caso B & $1,536 \mathrm{m3}$ \\
\hline Diferencia & $195 \mathrm{m3}$ \\
\hline$\%$ & $\mathbf{1 5 \%}$ \\
\hline
\end{tabular}

Fuente: Elaboración propia.

La cantidad de concreto requerida para el caso B es un $15 \%$ mayor que la requerida en el caso $A$. Dicho de otro modo, la influencia del concreto por superficie de entrepiso es de $0,15 \mathrm{~m} 3 / \mathrm{m}^{2}$ en el caso A, mientras que en el caso $B$ es de $0,18 \mathrm{~m} 3 / \mathrm{m}^{2}$.

Figura 4. Comparación de volumen de concreto entre casos

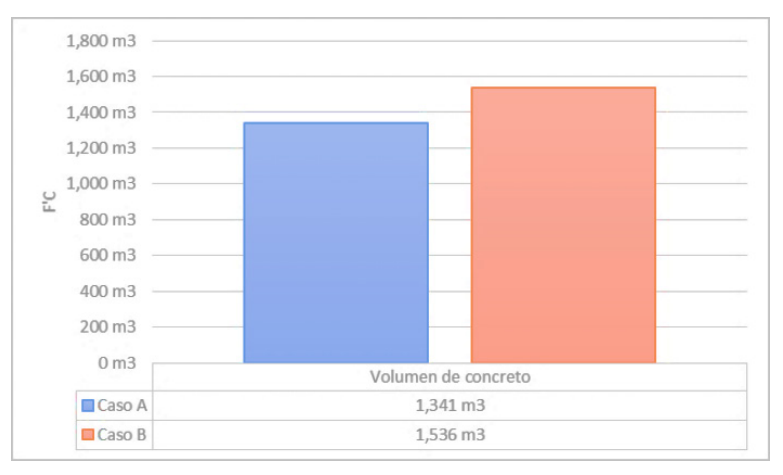

Fuente: Elaboración propia.

\section{Costo directo}

El factor económico es sin duda determinante al momento de definir entre sistemas constructivos. El costo directo, junto al programa de obra, son la base de la evaluación financiera y del análisis de inversión para cualquier proyecto.

Para el desarrollo del presente apartado, se investigan los costos de materias primas y rendimientos de mano de obra y equipos en el mercado de la ciudad de Bogotá y, a partir de este, se elaboró el análisis de precios unitarios (APU).

Una vez obtenido el valor por metro cuadrado para cada caso, se obtiene el valor total de la estructura a partir de la simple multiplicación de este por el área total de entrepiso para la edificación que es de $8,547 \mathrm{~m}^{2}$.

A continuación, se presenta el análisis de precios unitarios para el caso $A$, teniendo en cuenta que 
para este se tienen dos tipos de elementos prefabricados: losa alveolar para el entrepiso del cielo de sótano y losa maciza para el piso tipo.

Tabla 8. Costo directo total caso A

\begin{tabular}{|c|c|c|c|}
\hline Item & Área en planta & Valor / m2 & Valo Total \\
\hline Sótano & $3,277 \mathrm{~m} 2$ & $\$ 254,373$ & $\$ 833,623,189$ \\
\hline Piso 1 & $1,756 \mathrm{~m} 2$ & $\$ 212,900$ & $\$ 373,947,967$ \\
\hline Piso 2 & $1,756 \mathrm{~m} 2$ & $\$ 212,900$ & $\$ 373,947,967$ \\
\hline Piso 3 & $1,756 \mathrm{~m} 2$ & $\$ 212,900$ & $\$ 373,947,967$ \\
\hline \multicolumn{3}{|r|}{ Total } & $\$ 1,955,467,088$ \\
\hline
\end{tabular}

Fuente: Elaboración propia.

Dentro del el análisis de precios unitarios del caso $B$, se tendrán en cuenta tanto viguetas como loseta superior.

El índice de costo por $\mathrm{m}^{2}$ resulta de la relación del valor total de la estructura de entre el área total de la edificación.

Tabla 9. Diferencia del costo por entre $\mathrm{m}^{2}$ casos

\begin{tabular}{|c|c|c|c|}
\hline Caso & Area & Costo Total & Indice $\$$ por $\mathrm{m} 2$ \\
\hline Caso A & $8,547 \mathrm{~m} 2$ & $\$ 1,955,467,088$ & $228,803 \$ / m 2$ \\
\hline Caso B & $8,547 \mathrm{~m} 2$ & $\$ 1,569,778,754$ & $183,675 \$ / \mathrm{m} 2$ \\
\hline \multicolumn{3}{|r|}{ Diferencia } & $-45,128 \$ / m 2$ \\
\hline & & $\%$ & $-20 \%$ \\
\hline
\end{tabular}

Fuente: Elaboración propia.

El índice de costo por $\mathrm{m}^{2}$ del caso B es de un $20 \%$ menor frente al caso A.

Figura 5. Comparación del costo directo por $\mathrm{m}^{2}$.

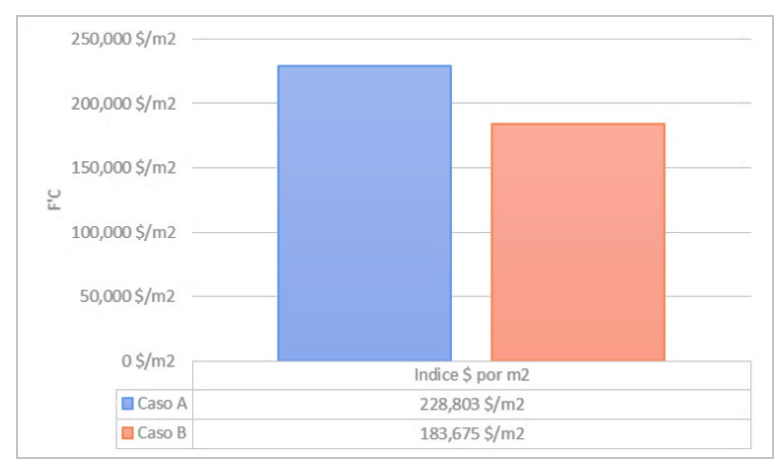

Fuente: Elaboración propia.

\section{Programa de obra}

El componente tiempo es una de las consideraciones más importantes dentro de un proyecto de construcción. La fecha final de entrega afecta los costos indirectos, pues una mayor duración de la obra aumenta los gastos generales y los gastos administrativos; por otro lado, posponer la puesta en servicio de una edificación puede implicar gastos adicionales o pérdidas económicas para inversionistas.

Los programas de obra se pueden diferenciar en programas de fabricación, transporte y montaje para el caso A y programas de construcción para el caso B.

Para el desarrollo de este componente, se acudió a fabricantes de prefabricados y a constructores con presencia en el mercado local. Se limita para ambos casos al análisis de los elementos de entrepiso. Los demás hitos, como actividades preliminares, columnas, vigas, acabados, etc., no se tendrán en cuenta.

La fabricación de elementos prefabricados corresponde a disponibilidad de pistas, moldes, ritmo de producción y requerimientos de fabricación de la empresa o planta de prefabricados. Así mismo, como se trata de una actividad que no se ejecuta dentro del proyecto, la responsabilidad de la obra se limita únicamente a la suficiente antelación de pedido o de orden de compra. Dicho lo anterior, no resulta comparable incluir la fabricación de las losas prefabricadas dentro del presente estudio.

Se parte de la suposición de que la estructura de vigas portantes sobre las que se instalarán las losas se encuentra terminada y lista para el montaje de los prefabricados. Se consideran 8 horas de trabajo diario y de acuerdo a las investigaciones con empresas prefabricadoras el rendimiento de instalación es de aproximadamente 15 losas al día. La anterior consideración es válida, siempre y cuando se trate de losas de las mismas dimensiones.

Para el caso A, en general, se tienen losas con idénticas dimensiones. Para el ejercicio de la duración de montaje en el presente caso, se consideran losas de medidas de 1,2 $\mathrm{m}$ de ancho por 7,5 $\mathrm{m}$ de longitud. 
Tabla 10. Duración de actividades caso A

\begin{tabular}{|c|c|c|c|c|c|}
\hline Item & $\begin{array}{c}\text { Horas trabajo } \\
\text { diario }\end{array}$ & $\begin{array}{c}\text { Rendimiento } \\
\text { diario (un) }\end{array}$ & $\begin{array}{c}\text { Rendimiento } \\
\text { diario }(\mathrm{m} 2)\end{array}$ & Área & $\begin{array}{l}\text { Duración total } \\
\text { (días hábiles) }\end{array}$ \\
\hline $\begin{array}{l}\text { Montaje losa } \\
\text { alveolar }\end{array}$ & $8 \mathrm{Hr} /$ día & 15 un & 135 m2/día & $3,277 \mathrm{~m} 2$ & 24 días \\
\hline $\begin{array}{l}\text { Montaje losa } \\
\text { maciza }\end{array}$ & $8 \mathrm{Hr} /$ día & 15 un & $135 \mathrm{~m} 2 /$ día & $5,269 \mathrm{~m} 2$ & 39 días \\
\hline $\begin{array}{l}\text { Capa de } \\
\text { compresión }\end{array}$ & $8 \mathrm{Hr} /$ día & & $120 \mathrm{~m} 2 /$ día & $8,547 \mathrm{~m} 2$ & 71 días \\
\hline \multicolumn{5}{|c|}{ Total elementos prefabricados } & 63 días \\
\hline & & & & Area total & $8,547 \mathrm{~m} 2$ \\
\hline \multicolumn{5}{|c|}{ Indice area instalada por día } & 135 m2/día \\
\hline
\end{tabular}

Fuente: Elaboración propia.

De acuerdo con lo anterior, la duración del montaje de los elementos prefabricados será de 63 días hábiles.

Teniendo en cuenta que los elementos prefabricados son pretensados, estos luego de ser ejecutados generan una contraflecha en su longitud, razón por la cual el vaciado de la capa de compresión se debe hacer inmediatamente se han instalado las losas prefabricadas, contemplándose este tiempo dentro de la duración de actividades del caso A mencionada en el párrafo anterior.

Con el caso B, luego de investigar el mercado local, se obtiene que el rendimiento de construcción de entrepiso con losa aligerada es de aproximadamente $500 \mathrm{~m}^{2}$ por semana, incluyendo el tiempo entre el vaciado de una losa y el encofrado de la losa del siguiente nivel. La anterior consideración aplica para entrepiso con una configuración en planta regular como es la que se tiene para el caso B.

Considerando 6 días de trabajo a la semana, a continuación, se presenta el análisis de duración de actividades para la losa de entrepiso tradicional.

Tabla 11. Duración actividades caso B

\begin{tabular}{|c|c|c|c|c|c|}
\hline Item & $\begin{array}{l}\text { Rendimiento } \\
\text { semana (m2) }\end{array}$ & $\begin{array}{c}\text { Dias trabajo } \\
\text { semanal }\end{array}$ & $\begin{array}{c}\text { Rendimiento } \\
\text { diario }(\mathrm{m} 2)\end{array}$ & Área & $\begin{array}{l}\text { Duración total } \\
\text { (días hábiles) }\end{array}$ \\
\hline Losa Sótano 1 & $500 \mathrm{~m} 2 / \mathrm{sem}$ & 6 días/sem & $83 \mathrm{~m} 2 /$ día & $3,277 \mathrm{~m} 2$ & 39 días \\
\hline Losa Tipo & $500 \mathrm{~m} 2 / \mathrm{sem}$ & 6 días $/$ sem & $83 \mathrm{~m} 2 /$ día & $5,269 \mathrm{~m} 2$ & 63 días \\
\hline \multicolumn{5}{|r|}{ Total } & 103 días \\
\hline \multicolumn{5}{|r|}{ Area total } & $8,547 \mathrm{~m} 2$ \\
\hline \multicolumn{5}{|c|}{ Indice area instalada por día } & $83 \mathrm{~m} 2 /$ día \\
\hline
\end{tabular}

Fuente: Elaboración propia.
La finalización de entrepiso en el caso B tarda 103 días, lo cual significa un $62 \%$ más que lo que demoraría la ejecución del entrepiso usando elementos prefabricados.

Tabla 12. Comparación duración actividades

\begin{tabular}{|r|r|c|}
\hline Caso & Area & Duración \\
\hline Caso A & $8,547 \mathrm{~m} 2$ & 63 días \\
\hline Caso B & $8,547 \mathrm{~m} 2$ & 103 días \\
\hline \multicolumn{2}{|r}{$\%$} & $62 \%$ \\
\hline
\end{tabular}

Fuente: Elaboración propia.

La anterior comparación se realizó con base en la suposición de que para ambos casos se tienen condiciones climáticas óptimas (tiempo seco) y aunque si bien es cierto que este factor no afecta la ejecución de actividades para el caso A, es importante aclararlo para mantener resultados comparables.

Figura 6. Duración de actividades para cada caso

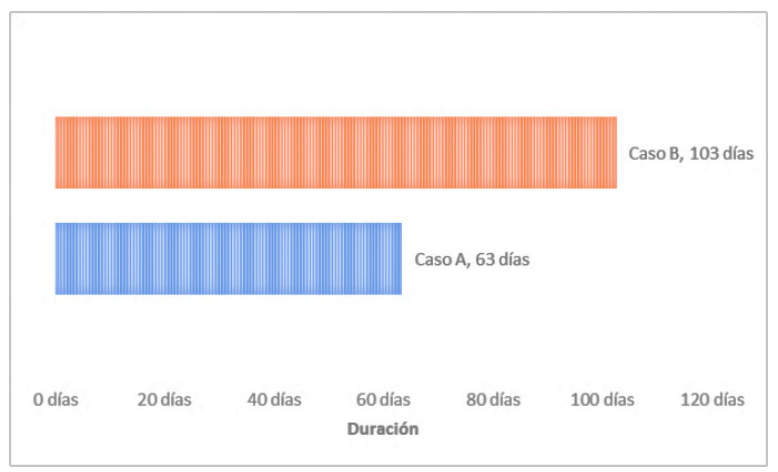

Fuente: Elaboración propia.

\section{Control de desperdicios}

Según datos del Green Building Council, las edificaciones emplean el $40 \%$ de los recursos no renovables (3 billones de toneladas al año) para construcciones alrededor del planeta [8].

Es ampliamente conocido que los componentes principales del concreto provienen de recursos no renovables. Con la explotación de canteras y el uso del agua se hace necesario tener procedimientos estrictos y responsables desde la elaboración hasta el vaciado del concreto y, de esta manera, aportar al logro de construcciones ambientalmente responsables. 
El desperdicio de material se define como el factor de pérdida mayor que la unidad que representa una mayor cantidad de concreto usado por razones del proceso constructivo, medios de vaciado, formaletas y moldes y experticia del personal operativo.

El porcentaje de desperdicio de concreto que se establece para elementos prefabricados es del $2 \%$, para el resto de elementos, capa de compresión (caso A) y viguetas y loseta superior (caso B), el desperdicio de material es del $5 \%$. Lo anterior representa una diferencia del $16 \%$ de mayor volumen final en el caso $B$ frente al caso $A$.

Tabla 13. Diferencia en porcentaje de desperdicio de material

\begin{tabular}{|c|c|c|c|c|}
\hline Caso & $\begin{array}{c}\text { Volumen de } \\
\text { concreto }\end{array}$ & $\begin{array}{c}\text { Porcentaje } \\
\text { desperdicio }\end{array}$ & $\begin{array}{c}\text { Volumen } \\
\text { Real }\end{array}$ & $\begin{array}{c}\text { Volumen } \\
\text { final }\end{array}$ \\
\hline Caso A - Prefabricados & $796 \mathrm{m3}$ & $2 \%$ & $812 \mathrm{~m} 3$ & \multirow{2}{*}{$1,384 \mathrm{~m} 3$} \\
\hline Caso A - Topping & $546 \mathrm{~m} 3$ & $5 \%$ & $573 \mathrm{m3}$ & \\
\hline Caso B - Viguetas & $852 \mathrm{~m} 3$ & $5 \%$ & $895 \mathrm{~m} 3$ & \multirow{2}{*}{$1,613 \mathrm{~m} 3$} \\
\hline Caso B - Loseta superio & $684 \mathrm{~m} 3$ & $5 \%$ & $718 \mathrm{~m} 3$ & \\
\hline \multicolumn{4}{|r|}{ Diferencia } & $228 \mathrm{~m} 3$ \\
\hline \multicolumn{4}{|r|}{$\%$} & $16 \%$ \\
\hline
\end{tabular}

Fuente: Elaboración propia.

Figura 7. Comparación volumen final de concreto en porcentaje de desperdicio

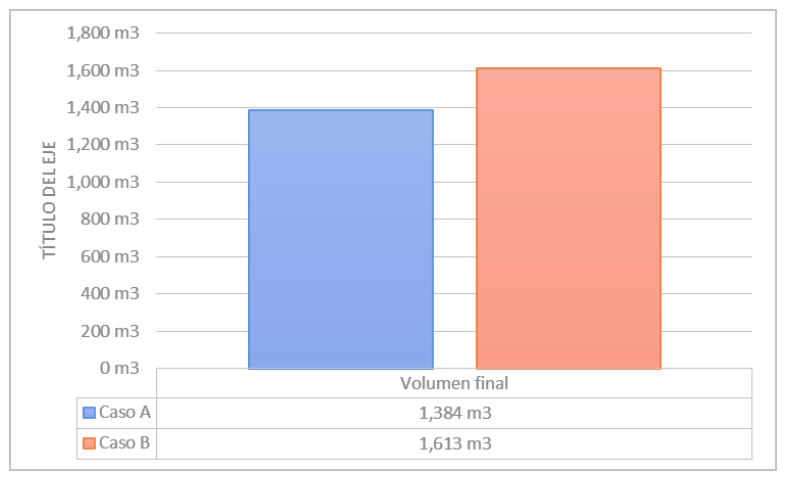

Fuente: Elaboración propia.

\section{Indicadores cuantitativos}

En el ámbito de las investigaciones universitarias es frecuente el uso de indicadores como herramienta de apoyo al autor o al evaluador de una investigación. Un indicador cuantitativo permite establecer diferencias entre dos o más elementos que se pretenden comparar [9].
A partir de los resultados obtenidos en el apartado anterior, continuación se describen y se generan para cada parámetro definido indicadores cuantitativos con el objetivo de generar herramientas que faciliten la toma de decisiones.

Indicador peso de la estructura - $I_{P E}$ Ante la ocurrencia de un sismo, entre más liviana sea una estructura, menor será la fuerza que tendrá que soportar. Además, una masa muy grande se mueve con mayor brusquedad y por lo tanto transmitirá mayor exigencia a sus elementos estructurales; por otro lado, entre menor sea el peso de la estructura, menores serán las cargas gravitacionales transmitidas a la cimentación, lo cual implica un menor costo de esta. Dicho lo anterior, a partir de la relación

$$
\frac{\text { Peso Caso } A}{\text { Peso Caso B }}
$$

Se establece que si el resultado de este cociente es menor que la unidad, se considera para el caso de estudio que el uso de elementos prefabricados representa una condición favorable para la losa de entrepiso de la edificación. Por otro lado, un resultado mayor que la unidad indicará como favorable la construcción del entrepiso haciendo uso métodos tradicionales, como el de una losa aligerada:

$$
I_{P E}=\frac{\text { Peso Caso } A}{\text { Peso } \operatorname{Caso} B}=\frac{3,219 T n}{3,686 T n}=0.87
$$

El valor $\mathrm{I}_{\mathrm{PE}}=087$, indica que, para el caso de estudio, el uso de componentes prefabricados resulta favorable para la edificación.

Indicador calidad de los materiales - $I_{C M}$

El uso de materiales con especificaciones exigentes tales como concretos de alto desempeño asegura la calidad y la alta resistencia de los elementos estructurales, lo que garantiza durabilidad al producto entregado y protege la inversión del usuario final de la edificación. La relación

$$
\frac{f^{\prime} c \operatorname{Caso} A}{f^{\prime} c \operatorname{Caso} B}
$$

Proporcionará la condición de favorable al caso A, si dicho cociente es mayor que la unidad. Un resultado contrario sucederá si el valor es menor que la unidad, pues este indicará que el caso $B$ resulta de ventaja dentro de la edificación.

La calidad del concreto de la capa de compresión (caso A) y de la loseta superior (caso B) es el mismo, 
por lo cual no se tendrán en cuenta estos elementos en la evaluación del indicador $\mathrm{I}_{\mathrm{CM}}$ :

$$
I_{C M=} \frac{f^{\prime} c \operatorname{Caso} A}{f^{\prime} c \operatorname{Caso} B}=\frac{40 M P a}{21 M P a}=1.90
$$

El valor $I_{C M}=1.90$, indica que, para el caso de estudio, el uso de componentes prefabricados resulta favorable para la edificación.

Indicador cantidad de concreto - $I_{c c}$ Mayor cantidad de material se traduce, en primer lugar, en mayor peso para la estructura. En segundo lugar, puede repercutir en mayor costo de la actividad $\mathrm{y}$, por último, obliga a la administración de mayor cantidad de material (controles de calidad, aseguramiento del recurso en el momento y cantidad requeridas, etc.). El resultado del cociente

$$
\frac{Q \operatorname{Caso} A}{Q \operatorname{Caso} B}
$$

Indica que si este es menor que la unidad, representa una condición favorable para el caso A, e igual condición para el caso $B$, si esta relación es mayor que la unidad:

$$
I_{C C=} \frac{Q \operatorname{Caso} A}{Q \operatorname{Caso} B}=\frac{1,341 m^{3}}{1,536 m^{3}}=0.87
$$

El valor $\mathrm{I}_{c c}=0.87$ indica que, para el caso de estudio, el uso de componentes prefabricados resulta favorable para la edificación.

Indicador costo directo - $I_{\text {APU }}$ A partir del análisis de precios unitarios elaborado para cada caso, y de la relación

$$
I_{A P U}=\frac{\text { Costo directo Caso } A}{\text { Costo directo Caso B }}
$$

Se define que si el indicador $\mathrm{I}_{\text {APU }}$ es menor que la unidad, representa una condición favorable para el caso $\mathrm{A}$, o condición positiva para el caso $\mathrm{B}$, si esta relación es mayor que la unidad:

$$
I_{A P U}=\frac{\text { Costo directo } \text { Caso } A}{\text { Costo directo Caso } B}=\frac{\$ 228,803 \mathrm{~m}^{2}}{\$ 183,675 \mathrm{~m}^{2}}=1.25
$$

Lo anterior indica que el índice lapu resulta favorable para el uso de sistemas tradicionales (in situ).

Indicador programa de obra - $I_{P O}$ Reducción de los cronogramas de obra significa, sin duda, ahorro de dinero tanto en el costo del proyecto como en recuperación de dinero por parte del inversionista. Así, resulta muy importante definir si el componente tiempo es favorable a una u otra solución. Dicho esto, para el indicador $I_{p O}$, resultante de la relación

$$
\text { Duración Caso A }
$$

Será favorable al caso A si su valor es menor que la unidad y favorable al caso B si su resultado es mayor que la unidad:

$$
I_{P O}=\frac{\text { Duración Caso A }}{\text { Duración Caso B }}=\frac{63 \text { días }}{103 \text { días }}=0.62
$$

Así entonces, en el componente tiempo, el uso de elementos prefabricados resulta de ventaja frente al empleo de la construcción tradicional.

Indicador sustentabilidad (control de desperdicios) - $I_{C D}$

El concepto de sustentabilidad está en algunos casos, ligado a un mayor costo, o por lo menos en lo que se refiere al costo directo de la obra. Sin embargo, ante la problemática ambiental que vivimos y que es mundialmente conocida, el ingeniero debe eliminar de su ejercicio el uso de prácticas que sigan haciendo uso indiscriminado de los recursos naturales de los que dispone.

Para el presente documento, el indicador sostenibilidad se basa en el porcentaje de desperdicios de concreto que genere la aplicación de sistemas prefabricados frente a sistemas in situ.

El indicador resultará de la relación

$$
\frac{Q_{\text {final }} \text { Caso } A}{Q_{\text {final }} \text { Caso B }}
$$

Siendo favorable al caso A si esta resulta menor que la unidad y favorable al caso B si su resultado es mayor que la unidad:

$$
I_{C D}=\frac{Q_{\text {final }} \operatorname{Caso} A}{Q_{\text {final }} \operatorname{Caso} B}=\frac{1,384 m^{3}}{1,613 m^{3}}=0.85
$$

Teniendo en cuenta el indicador $\mathrm{I}_{C D}=0.85$, usar elementos prefabricados resulta favorable no solo frente a los sistemas tradicionales, sino también favorable a la construcción de edificaciones ambientalmente responsables.

\section{Consolidación de indicadores}

Se muestra a continuación una tabla resumen con la consolidación de los indicadores evaluados. 
Tabla 14. Consolidación de indicadores

\begin{tabular}{|c|c|c|c|}
\hline Indicador & $\begin{array}{c}\text { \% de } \\
\text { importancia }\end{array}$ & Valor & Condición \\
\hline $\mathrm{I}_{\mathrm{PE}}$ & $20.0 \%$ & 0.87 & Favorable Caso $\mathrm{A}$ \\
\hline $\mathrm{I}_{\mathrm{CM}}$ & $13.3 \%$ & 1.90 & Favorable Caso A \\
\hline $\mathrm{I}_{\mathrm{CC}}$ & $13.3 \%$ & 0.87 & Favorable Caso $\mathrm{A}$ \\
\hline $\mathrm{I}_{\mathrm{APU}}$ & $20.0 \%$ & 1.25 & Favorable Caso B \\
\hline $\mathrm{I}_{\mathrm{PO}}$ & $20.0 \%$ & 0.62 & Favorable Caso A \\
\hline $\mathrm{I}_{\mathrm{CD}}$ & $13.3 \%$ & 0.85 & Favorable Caso A \\
\hline
\end{tabular}

Fuente: Elaboración propia.

Como se observa, de los 6 indicadores analizados y para la escala establecida, el uso de sistemas prefabricados representa el $80 \%$ de la escala de importancia y el $20 \%$ restante lo representan los sistemas in situ. De lo anterior, se puede concluir como positivo el uso de elementos prefabricados frente al uso de sistemas constructivos tradicionales.

\section{CONCLUSIONES}

Los procesos de construcción para losas de entrepiso son, sin duda, diferentes entre ambos sistemas. Los métodos in situ, a pesar de los avances en tecnología del concreto, siguen siendo artesanales, lo que da espacio a errores por factores humanos y mantenie incertidumbre en el cumplimiento de plazos de construcción. Por otro lado, los prefabricados se ejecutan en fábricas con estrictos parámetros de calidad, con procesos industrializados que finalizan con actividades de transporte y montaje con requerimientos de mano de obra y equipos mínimos.

Los indicadores del caso de estudio que resultaron de la presente investigación permiten establecer cuál sistema para losas de entrepiso resulta de mayor ventaja entre uno u otro. Además, proporcionan en términos de orden de magnitud la relación de cada aspecto analizado frente a cada sistema, si se quisieran evaluar por separado.

El resultado de la evaluación de los indicadores cuantitativos de comparación (peso de la estructura $-I_{P E}-$, calidad de los materiales $-I_{C M}$, cantidad de concreto $-\mathrm{I}_{c C}-$, programa de obra $-\mathrm{I}_{\mathrm{PO}}-\mathrm{y}$ el indicador de sustentabilidad $-\mathrm{I}_{\mathrm{CD}}-$ ) permiten establecer que el uso de elementos prefabricados en losas de entrepiso es una solución óptima en la construcción de losas de entrepiso. Únicamente la evaluación del indicador de Costo - $\mathrm{I}_{\mathrm{APU}}$ - concluye que el uso de sistemas in situ es favorable frente al uso de prefabricados.
Con los criterios evaluados, se evidencia que el uso de elementos prefabricados en la construcción de losas de entrepiso es ampliamente más favorable que el uso de sistemas de losas aligeradas. Si bien es cierto que han sido pocas las investigaciones y estudios de la temática de esta investigación, los resultados en cuanto a mejoramiento de diseños, calidad de las construcciones, cumplimiento de plazos y aporte a las sustentabilidad obtenidos en el presente documento suponen un panorama positivo a la aplicación en la construcción de elementos prefabricados.

El país cuenta con los medios para ejecutar construcciones prefabricadas, existen empresas en el mercado intentando hacerse un lugar, garantizando tecnología, calidad, presupuestos cerrados y cumplimiento de plazos. Haría falta más divulgación y el ajuste de las normas vigentes, dándole lugar al prefabricado como un elemento tan confiable como los que se utilizan en la actualidad.

\section{REFERENCIAS}

[1] Caamacol, "Noticias” [En línea]. 2014. Disponible en: https://camacol.co/noticias/innovación-clave-para-la-competitividad-del-sector-de-la-construcción

[2] Camacol, “Expocamacol”. Medellín: 2016.

[3] L. S. Pheng y C. J. Chuan, "Just-In Time Mangement of Precast Concrete Components", Journal of Construction Engineering and Management, vol. 127, 2001.

[4] L. Koskela, "Is structural change the primary solution to the problems of construction?", Journal Building Research \& Information, 2003, pp. 85-96.

[5] C. Pasquire, A. Gibb y N. Blismas, "What Should You Really Measure If You Want to Compare Pre-fabrication with Traditional Constructio"n, 13th International Group for Lean Construction Conference: Proceedings. Sydney: International Group on Lean Construction. Sidney: 2005.

[6] W. Rodríguez, Gerencia de construcción y del tiempo-costo, Lima: Macro EIRL, 2013.

[7] M. Reguant y M. Torrado, El método Delphi. Barcelona: Reire, 2016. 
[8] U.S. Green Building Council (USGBC), An Introduction to the U.S. Green Building Council and the Leed Green Building Rating System. Washington: 2005.
[9] A. López, M. Valcárce y M. Barbancho, “Indicadores cuantitativos y cualitativos para la evaluación de la actividad investigadora: ¿Complementarios? ¿Contradictorios? ¿Excluyentes?”. [En línea] 2005. 